\title{
A comparison study of post-operative infection analysis of cold-knife conization and loop electrosurgical excision procedure for cervical high-grade squamous intraepithelial lesion
}

\author{
Liangzhi Cai ${ }^{1}$, Yunmei Huang ${ }^{2}$, Chaoqin Lin ${ }^{1}$, Guifeng Liu ${ }^{1}$, Xiaodan Mao ${ }^{3}$, Binghua Dong ${ }^{3}$, Ting Lu$^{4}$, \\ Pengming Sun ${ }^{1,3}$ \\ ${ }^{1}$ Department of Gynaecology, Fujian Provincial Maternity and Children's Hospital, Affiliated Hospital of Fujian Medical University, Fuzhou 350001, China; \\ ${ }^{2}$ Academy of Integrative Medicine, Fujian University of Traditional Chinese Medicine, Fuzhou 350122, China; ${ }^{3}$ Laboratory of Gynaecologic Oncology, \\ ${ }^{4}$ Department of Pathology, Fujian Provincial Maternity and Children's Hospital, Affiliated Hospital of Fujian Medical University, Fuzhou 350001, China \\ Contributions: (I) Conception and design: L Cai; (II) Administrative support: C Lin, P Sun; (III) Provision of study materials or patients: C Lin, T Lu; \\ (IV) Collection and assembly of data: G Liu, X Mao, B Dong; (V) Data analysis and interpretation: L Cai, Y Huang; (VI) Manuscript writing: All \\ authors; (VII) Final approval of manuscript: All authors. \\ Correspondence to: Prof. Dr. Pengming Sun, PhD, MD. Laboratory of Gynaecologic Oncology, Fujian Provincial Maternity and Children's Hospital, \\ Affiliated Hospital of Fujian Medical University, Fuzhou 350001, China. Email: sunfemy@hotmail.com.
}

Background: High-grade squamous intraepithelial lesion (HSIL) is a premalignant condition of the cervical cancer. Loop electrosurgical excision procedure (LEEP) and cold-knife conization (CKC) were the most effective treatment. Most studies focused on the recurrence rate, positive margin rate, residual disease rate, secondary hemorrhage or cervical stenosis of these two methods. At present, there are few researches about the post-operative infection comparing LEEP with CKC for treating HSIL.

Methods: One hundred and fourteen patients diagnosed as HSIL were underwent cold conization $(n=43)$ or LEEP ( $\mathrm{n}=71$ ), according to 1:2 matching approximately and being divided randomly into two groups. The information, including the post-operational inflammatory complications, the leucorrhea abnormalities, the pathogens isolated from cervical secretions and pathological reports, were collected for comparison.

Results: There was no significant difference between them in bleeding, diameter, depth or volume of tissue between two groups. However, the operation time of the CKC group $(24.81 \pm 11.09)$ minutes was longer than that of LEEP group $(15.79 \pm 9.82)$ minutes. Seventeen patients of the LEEP group were admitted postoperatively as emergencies for secondary-hemorrhage. But it did not happen in CKC group. During the follow-up period, 28 patients (CKC $8 v s$. LEEP 20) were diagnosis as reproductive tract infections. Fourteen patients (CKC 6 vs. LEEP 8) had leucorrhea abnormalities. Eighteen patient (CKC 3 vs. LEEP 15) isolated pathogens from their cervical secretions. There was no significant correlation between leucorrhea abnormality and cervical secretion abnormality. The positive rate of cervical secretion infection in the LEEP group was higher than the CKC group $(\mathrm{P}<0.05)$. The inflammatory response and process had some pathological difference in post-operation time of two groups, especially in those secondary hemorrhage cases.

Conclusions: These two excision procedures for treating HSIL have their respective advantages and disadvantages. This study indicates that the incidence of persistent cervical infection after the CKC operation with Sturmdorf suturing is lower than that of after LEEP surgery. Clinicians should pay attention to the pathogen isolated from cervical post-operative secretion. It is conducive to find hidden pathogens and control subsequent surgical inflammation.

Keywords: High-grade squamous intraepithelial lesion (HSIL); loop electrosurgical excision procedure (LEEP); cold-knife conization (CKC); cultured pathogens

Submitted Aug 06, 2019. Accepted for publication Nov 29, 2019.

doi: $10.21037 /$ tcr.2019.12.34

View this article at: http://dx.doi.org/10.21037/tcr.2019.12.34 


\section{Introduction}

High-grade squamous intraepithelial lesion (HSIL) is a premalignant condition of the cervical cancer. Patients suffering from HSIL can be diagnosed by a three-step screening. To prevent the cancer foundation, surgical excision is usually carried out especially in high-grade lesions (1). This way was also prude to be effective. As a traditional procedure for HSIL, cold-knife conization (CKC) is typically performed in hospitals. While in 1989, Prendiville (2) adapted a new excision procedure-loop electrosurgical excision procedure (LEEP) to do the treatment. Wright and Lindeque described LEEP was an effective method for evaluation and treatment of $\operatorname{HSIL}(3,4)$. These recommended treatments are two main methods of excision for high grade lesion with their respective advantages and disadvantages (4,5). Many researchers (6-9) compared LEEP with CKC in HSIL focusing on the recurrence rate, positive margin rate, residual disease rate, secondary hemorrhage or cervical stenosis. Their data suggested that LEEP is as effective as CKC in HSIL treatment according to the recurrence rate, positive margin rate, residual disease rate, secondary hemorrhage, and cervical stenosis $(10,11)$.

Meanwhile, there are patients who suffered from cervical inflammatory disease after surgical excision. It was reported that the genital infection rate after CKC was $36 \%$ (12). In comparison, the genital infection rate after LEEP was ranging from $0.8 \%$ to $14.4 \%(8,13-19)$. But in these studies, few scholars have proposed the criteria to define postoperative infection and the follow-up time for infection was only 2-3 weeks or even not mentioned. As known, these infections were probably due to the pathogens in the cervix. Hysterectomy is not a primary treatment for HSIL. Furthermore, occult invasive cervical cancer could be found by the pathological result. Some of them would need a following hysterectomy. Simple hysterectomy may compromise treatment outcomes for the patient (5). The infections would delay the time of hysterectomy. Which method can reduce the cervical inflammation? In considering of postoperative cervical inflammation, such as edema and congestion, when is the appropriate interval time to do the hysterectomy ?

In this study, the information of leucorrhea abnormality, pathogen isolated from cervical secretions, the pathological analysis were collect in HSIL patients after CKC or LEEP. The outcome from different angle was to compare the different cervical conization procedures for HSIL, to reveal the pathogens change in cervical secretions after cervical conization. The pathological analysis was taken to evaluate the procedure and show the inflammatory response during the follow-up. The results of the study can help to reveal the duration of inflammation, the type of susceptible pathogen and the time of susceptibility, which can provide the basis for choosing the best interval time for the subsequent surgical treatments and selecting the corresponding perioperative antibiotics.

\section{Methods}

\section{Clinical samples}

This study was conducted on the 120 patients who underwent colposcopy in Fujian Province Women and Children's Health Hospital during 2015-2017. The number of cases between the two groups was divided randomly into two groups, according to 1:2 matching approximately. Six of them missed follow-up during the period. Women who underwent CKC were recruited into the CKC group $(n=43)$, and the other the same aged patients were recruited in the groups of LEEP $(n=71)$. All above patients underwent colposcopic evaluation and were pathologically diagnosed as HSIL by cervical biopsy-confirmed. All patients were given general anesthesia and studied in the lithotomy position. Eligible women were not pregnant or had not been pregnant in the past 6 months; had no previous surgery, dilation and curettage procedure, or abortive outcomes; and had no cancer, HIV/AIDS or autoimmune disease. At recruitment, informed written consent was obtained from each woman. All protocols and informed consent procedures were approved by the ethics committee of the hospital. First, cervical transformation was discriminated by application of Lugol's solution. CKC was done by scalpel, and conical tissue that extended to the endocervical area was removed. After the removal, the Sturmdorf method was used to suture the residue of the uterine cervix. For the LEEP, a loop electrode was fitted into the cervix to remove conical tissue containing the transformation zone and some part of the endocervical canal from the cervix. All the surgical excised tissues were performed using Pathological analysis.

The patients were followed-up at 2, 4, 8, 12 postoperation weeks. Doctors exposed the cervix by the use of a vaginal speculum. A sterile swab with physiological brine was introduced to the cervical surface. Secretions were collected by cotton swabs within $1.5-2 \mathrm{~cm}$ (remaining for $30 \mathrm{~s}$ ) to obtain pathogens in cervical canal and used for cultivation 
Table 1 Comparison of characteristics of the CKC and the LEEP groups

\begin{tabular}{|c|c|c|c|}
\hline Characteristics & $\begin{array}{c}\text { CKC }(n=43) \\
n(\%)\end{array}$ & $\begin{array}{l}\text { LEEP }(\mathrm{n}=71) \\
\mathrm{n}(\%)\end{array}$ & $P$ value \\
\hline Age (years) & $48.95 \pm 7.68$ & $46.66 \pm 7.07$ & $0.43^{\mathrm{a}}$ \\
\hline $\mathrm{n} \leq 30$ & $\mathrm{~N} / \mathrm{A}$ & $1(1.40)$ & $0.74^{\mathrm{b}}$ \\
\hline $30<n \leq 40$ & $5(11.63)$ & $9(12.68)$ & \\
\hline $40<\mathrm{n} \leq 50$ & $20(46.51)$ & $37(52.11)$ & \\
\hline$n>50$ & $18(41.86)$ & $24(33.80)$ & \\
\hline Smoker & $2(4.65)$ & $5(7.04)$ & 0.61 \\
\hline \multicolumn{2}{|c|}{$\begin{array}{l}\text { History of gestation } \\
\text { (including delivery and abortion) }\end{array}$} & & 0.87 \\
\hline 0 & $2(4.65)$ & $5(7.04)$ & \\
\hline 1 & $10(23.26)$ & 14 (19.72) & \\
\hline 2 & $19(44.19)$ & 35 (49.29) & \\
\hline$>2$ & $12(27.91)$ & $17(23.94)$ & \\
\hline Mode of delivery $(n=107)$ & 41 & 66 & 0.84 \\
\hline Vagina & $24(58.54)$ & $37(56.06)$ & \\
\hline Caesarean & $11(26.83)$ & 21 (31.82) & \\
\hline Both & $6(14.63)$ & $8(12.12)$ & \\
\hline $\begin{array}{l}\text { Infection risk of } \\
\text { complications (\%) }\end{array}$ & 11.63 & 8.45 & 0.40 \\
\hline $\begin{array}{l}\text { Interval time between } \\
\text { excisional treatment and } \\
\text { following surgery (days) }\end{array}$ & $32.86 \pm 15.46$ & $43.32 \pm 15.17$ & 0.62 \\
\hline
\end{tabular}

${ }^{a}$, indicates that there is no significant difference in the mean $\pm S D$ of age between two groups. ${ }^{b}$, indicates that there is no significant difference in the age groups between two groups. CKC, coldknife conization; LEEP, loop electrosurgical excision procedure.

of bacteria, drench coccus, mycoplasma, fungi, anaerobic bacteria, chlamydia, germiculture and chlamydia detection.

\section{Pathogen culture and identification}

Columbia blood agar was used as selective medium to isolate viable aerobic. Chocolate blood agar plate medium was used for Neisseria gonorrboeae. Sabouraud glucose liquid medium was used for Fungi. These specimens were cultured in an incubator for $24 \mathrm{~h}$ and suspicious colonies were selected and identified according to the bioMerieux Vitek 32 microbial identification system. Anaerobic bacteria identification specimens were placed in bags for 24-48 h.
Suspicious colonies were selected for the oxygen resistance test. All these media were purchased from Bioivd Biological Technology (Zhengzhou) Co., LTD. Kit of Rachanda Biological Technology (Shanghai) Co., LTD. was used for mycoplasma culture identification according to their instructions. Kit of Chlamydia trachomatis Antigen made in Britain was used for the chlamydia detection according to the specifications.

\section{Pathological analysis}

All pathological specimens were examined and diagnosed by two experienced pathologists. According to the pathological reports, patients' wills and the follow-up condition, some of them needed a repeat conization or following hysterectomy. The surgical excised tissues were fixed in formalin, paraffinembedded, and prepared as 5 - $\mu \mathrm{m}$-thin paraffin sections. The sections were stained with hematoxylin-eosin stain, observed, and analyzed under a microscope.

\section{Statistical analysis}

The data were entered into the EpiData software v3.1 (EpiData Association, Odense, Denmark) and were analyzed using IBM SPSS Statistics (Version 24.0, IBM, USA). Continuous data were expressed as mean \pm standard deviation, and categorical data were reported as percentages. The Student's $t$-test was used to compare continuous parametric variables. The Pearson's Chi-square or Fisher's exact test were used to compare the proportion of target outcomes. A $\mathrm{P}$ value less than 0.05 was considered statistically significant.

\section{Results}

\section{Comparison of characteristics of the CKC and the LEEP groups}

One hundred and fourteen women were reviewed. Mean age was 48.95 years old (range, 39-68 years old, $n=43$ ) in CKC group, while mean age was 46.66-year-old (range, 31-64 years old, $\mathrm{n}=71$ ) in LEEP group. Comparison of characteristics revealed no significant difference between the two groups, including age, smoker, history of gestation, mode of delivery, infection risk of complications (for example diabetes, anemia, immune defection, and malnutrition), and interval time between excisional treatment and following surgery $(\mathrm{P}>0.05)$ (Table 1). 
Table 2 Comparison of operation characters of the CKC and the LEEP groups

\begin{tabular}{|c|c|c|c|}
\hline Operation characters & CKC $(n=43)$ & LEEP $(n=71)$ & $P$ value ${ }^{a}$ \\
\hline Operation time (minutes) & $24.81 \pm 11.09$ & $15.79 \pm 9.82$ & $<0.001$ \\
\hline Bleeding (mL) & $7.08 \pm 9.82$ & $7.12 \pm 6.12$ & 0.98 \\
\hline Diameter of tissue $(\mathrm{mm})$ & $2.43 \pm 0.52$ & $2.50 \pm 0.43$ & 0.53 \\
\hline Depth of tissue (mm) & $1.87 \pm 0.41$ & $1.92 \pm 0.47$ & 0.63 \\
\hline Volume of tissue $\left(\mathrm{mm}^{3}\right)$ & $3.15 \pm 1.83$ & $3.26 \pm 1.82$ & 0.77 \\
\hline $\begin{array}{l}\text { Cases of further treatment, } \\
n(\%)\end{array}$ & $9(20.93)$ & $16(22.54)$ & 0.84 \\
\hline Repeat conization & $3(6.98)$ & $12(16.90)$ & \\
\hline Hysterectomy & $6(13.95)$ & $4(5.63)$ & \\
\hline
\end{tabular}

${ }^{a}$, P value $<0.05$ indicates significant difference. CKC, cold-knife conization; LEEP, loop electrosurgical excision procedure.

Table 3 Comparison of outcomes of the CKC and the LEEP groups

\begin{tabular}{|c|c|c|c|}
\hline Outcomes & CKC $(n=43)$ & LEEP $(n=71)$ & $P$ value ${ }^{a}$ \\
\hline Secondary hemorrhage (\%) & $\mathrm{N} / \mathrm{A}$ & $17(23.94)$ & $<0.001$ \\
\hline$<2$ weeks & N/A & 14 & \\
\hline 2-4 weeks & N/A & 3 & \\
\hline $4-8$ weeks & N/A & $\mathrm{N} / \mathrm{A}$ & \\
\hline 8-12 weeks & N/A & $\mathrm{N} / \mathrm{A}$ & \\
\hline $\begin{array}{l}\text { Reproductive tract } \\
\text { infections (\%) }\end{array}$ & $8(18.60)$ & $20(28.17)$ & 0.18 \\
\hline $\begin{array}{l}\text { Leucorrhea abnormalities } \\
\text { (\%) }\end{array}$ & $6(13.95)$ & $8(11.27)$ & 0.44 \\
\hline$<2$ weeks & $\mathrm{N} / \mathrm{A}$ & $\mathrm{N} / \mathrm{A}$ & \\
\hline 2-4 weeks & 5 & 5 & \\
\hline $4-8$ weeks & 1 & 3 & \\
\hline 8-12 weeks & $\mathrm{N} / \mathrm{A}$ & $\mathrm{N} / \mathrm{A}$ & \\
\hline $\begin{array}{l}\text { Positive rate of pathogens } \\
\text { isolated from cervical } \\
\text { secretions (\%) }\end{array}$ & $3(6.98)$ & $15(21.13)$ & 0.04 \\
\hline$<2$ weeks & 1 & N/A & \\
\hline 2-4 weeks & 1 & 10 & \\
\hline $4-8$ weeks & 1 & 5 & \\
\hline 8-12 weeks & N/A & $\mathrm{N} / \mathrm{A}$ & \\
\hline
\end{tabular}

a,$P$ value $<0.05$ indicates significant difference. $C K C$, cold-knife conization; LEEP, loop electrosurgical excision procedure.

\section{Comparison of operation characters of the CKC and the LEEP groups}

The operation characters of the two groups, including operation time, bleeding, diameter, depth and volume of tissues, and cases of further treatment were investigated. The operation time of the CKC group $(24.81 \pm 11.09 \mathrm{~min})$ was much longer than that of LEEP group $(15.79 \pm 9.82 \mathrm{~min})$ $(\mathrm{P}<0.01)$. However, there was no significant difference between them in bleeding, diameter, depth or volume of tissue $(\mathrm{P}>0.05)$. And 25 cases need further treatment, 3 patients of CKC group had positive margin and 12 women of LEEP group had positive margin, so they need repeat conization. According to the pathological reports, 10 patients suffered from cervical intraepithelial neoplasia III, because of their poor follow-up condition, they want a hysterectomy. Six in CKC group, and 4 in LEEP group (Table 2).

\section{Comparison of outcomes of the CKC and the LEEP groups}

Seventeen patients of the LEEP group were admitted postoperatively as emergencies for secondaryhemorrhage. None of the patients were admitted within 24 hours after the operation. Fourteen $(82.35 \%)$ of them were admitted in 10-14 days after the operation. Three $(17.65 \%)$ of them were admitted in 2-4 weeks after the operation (Table 3). All of them were filled the gauze in the vaginal to oppress hemostasis for 24 hours. However, 4 cases of treatments were not so good because of repeat bleeding and they were treated in the emergency room under local anesthesia by electrocoagulation/fulguration with ball electrodes. One case was treated under general anesthesia and underwent suturing of the cervix. But it did not happen in the CKC group.

Two groups of reproductive tract infections, the relation between leucorrhea and cervical abnormalities had no significant difference. During the follow-up period, 28 (CKC $8 v$ s. LEEP 20) patients were diagnosis as reproductive tract infections. Fourteen patients had leucorrhea abnormalities. Six patients in CKC group had leucorrhea abnormalities, while 8 patients in LEEP group had leucorrhea abnormalities. Eighteen patients isolated pathogens from their cervical secretions. Three patients in CKC group had pathogens isolated from cervical secretions, while 15 patients of LEEP group culture pathogens from cervical secretions. There was no significant correlation between leucorrhea abnormalities and cervical abnormalities $(\mathrm{P}>0.05)$. There 
Table 4 Pathogens cultured from cervical secretion

\begin{tabular}{lcc}
\hline Pathogen & $\mathrm{CKC}(\mathrm{n}=43)$ & $\operatorname{LEEP}(\mathrm{n}=71)$ \\
\hline Gram-negative bacteria & 1 & 6 \\
E. coli & $\mathrm{N} / \mathrm{A}$ & 1 \\
K. pneumoniae & $\mathrm{N} / \mathrm{A}$ & 1 \\
B. fragilis & $\mathrm{N} / \mathrm{A}$ & 1 \\
Gram-positive bacteria & 1 & $\mathrm{~N} / \mathrm{A}$ \\
E. faecalis & $\mathrm{N} / \mathrm{A}$ & 1 \\
S. aureus & & 2 \\
S. anginosus & 1 & 1 \\
Urealyticum & & 2 \\
U. urealyticum & $\mathrm{N} / \mathrm{A}$ & 15 \\
Fungi & $\mathrm{N} / \mathrm{A}$ & 3 \\
M. albicans & 3 & \\
T. glabrata & & \\
Total & &
\end{tabular}

CKC, cold-knife conization; LEEP, loop electrosurgical excision procedure.

were only 4 leucorrhea abnormal patients had pathogens isolated from cervical secretions. However, the positive rate of cervical secretions in the LEEP group was significantly higher than in the CKC group (Tables 3 and 4), and the difference was most significant at the time between 2 and 8 weeks after operation $(\mathrm{P}<0.05)$.

The leucorrhea abnormalities of CKC group were usually concentrated between 2 and 4 weeks. Other time was seldom. There were 3 cases had pathogens isolated from cervical secretions, 1 case was in 2 weeks, 1 case was between 2 and 4 weeks, 1 case was in between 4 and 8 weeks. While in the LEEP group, there were 5 cases of the leucorrhea abnormalities between 2 and 4 weeks. Three cases were between 4 and 8 weeks. In the incidence of inflammation, the positive pathogen culture of cervical secretion showed, there were 10 cases between 2 and 4 weeks and 5 cases were between 4 and 8 weeks. The bacterial infection in the CKC group was not specific, but the LEEP group was mainly $E$ coli infection. $K$ pneumoniae, $U$ urealyticum, and $E$ faecalis were also found.

\section{The result of pathological analysis}

It was found that both the CKC and LEEP groups showed the different healing condition. In the first two weeks after the operation, the CKC group showed an acute inflammation with increased neutrophils, tissue edema, and capillary congestion. The LEEP group marginal tissue had severe carbonation with protein degeneration. The cervix also had acute inflammatory reactions. Two and four weeks after the operation, tissue inflammation in the CKC group decreased to chronic condition, but in the LEEP group, the cervical eschar began to fall off, especially in the cases of secondary hemorrhage. The pathology manifestation of tissue showed edema, bleeding and inflammatory cells infiltration. Four weeks post-operation, the CKC showed chronic inflammatory manifestations with lymphocyte growth and corresponding tissue proliferation. The tissue of LEEP group had acute inflammatory reaction prolonged at the same time, especially the cases of secondary hemorrhage. More than 8 weeks after the surgery, both groups showed chronic inflammatory manifestations, with lymphocyte growth and corresponding tissue proliferation (Figure 1).

\section{Discussion}

The advantages and disadvantages of the two excisional treatments have been studied and analyzed by many researchers. Some studies suggested that LEEP is a shorter procedure. And it causes less intra-operative bleeding and results in a shorter hospital stay. Whereas pathologists believe that LEEP cones are shallower with overall less volume and weight than CKC. In addition, it is difficult to make an accurate judgment about whether there is residual HSIL tissue remaining on the edge of the excision due to the carbonization zone of LEEP, which can be thermally damaged. This needs more follow-up investigation to be clarified. There are no similar problems of the cervical cone cutting edge of CKC. Due to thorough suturing and ligation, distant postoperative bleeding caused by crusts falling is precluded. Furthermore, it is easier to identify whether there is any residue, which provides a concrete basis for whether supplemental conization is needed.

Some studies showed that the healing time of cervical operation is more than 6 weeks and the incidence of infectious complication is up to more than $30 \%(18,19)$. Song et al. proved that $90 \%$ of the effective length of the cervix was regenerated 6 months post-operation (20). It could be clearly seen that the eversion of the endocervical mucosa during the follow-up. These patients often went back to see the doctor because of cervical contact 


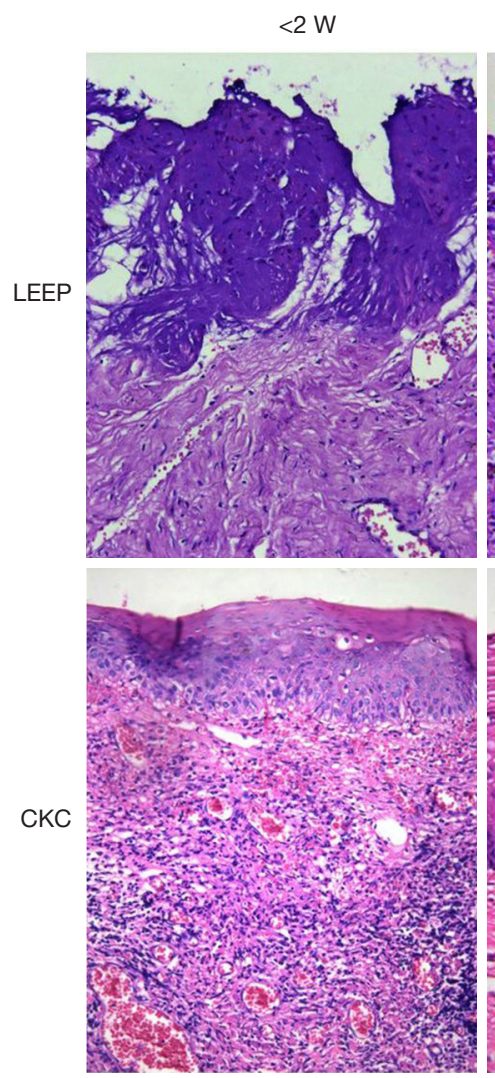

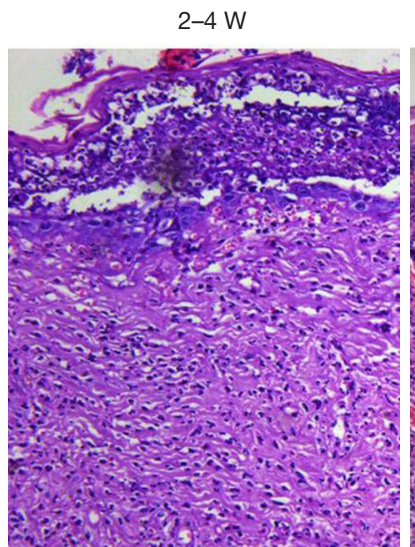
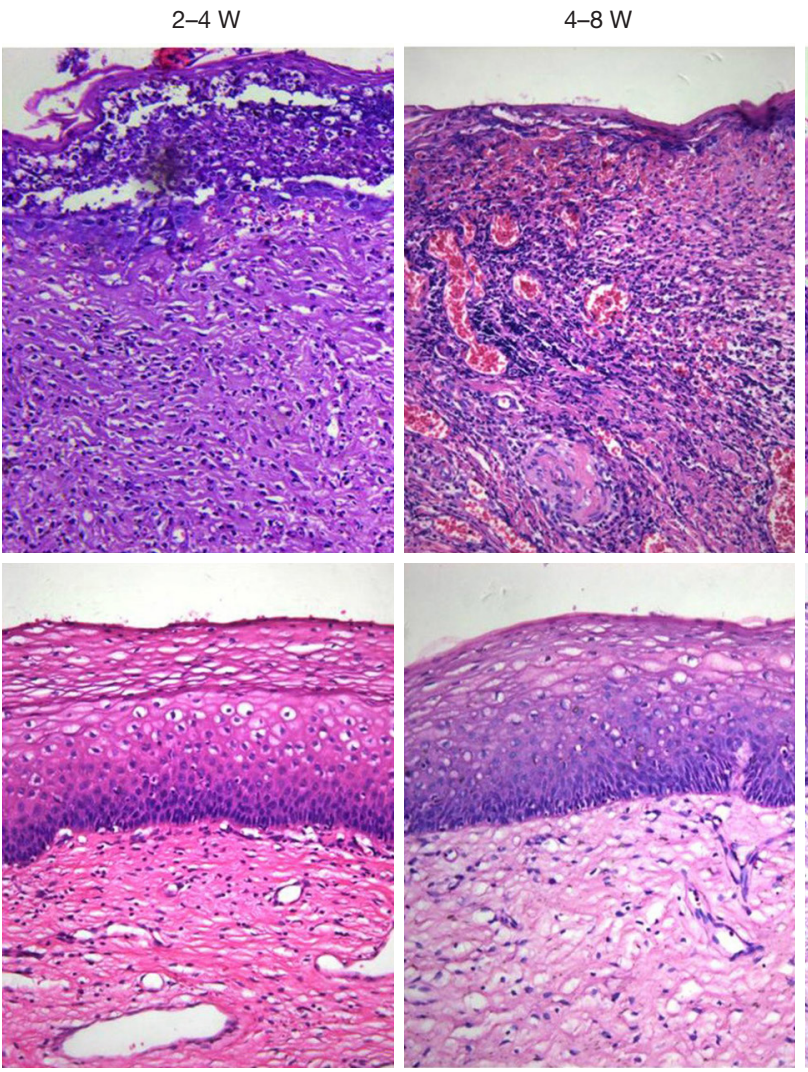
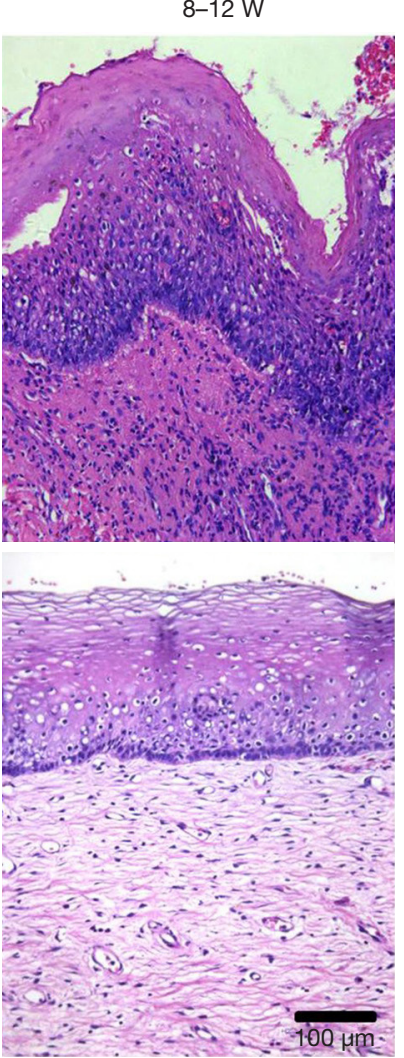

Figure 1 The pathological tissue sections were applied with hematoxylin-eosin staining. In the first two weeks, the CKC group showed an acute inflammation with increased neutrophils, tissue edema, and capillary congestion. The LEEP group marginal tissue had severe carbonation and acute inflammatory reactions. Two and four weeks after the operation, the inflammation response in CKC group were obviously relieved, but the LEEP group showed bleeding and inflammatory cells infiltration due to the eschar falling off. In between four and eight weeks, the CKC group showed chronic inflammatory manifestations with lymphocyte growth and corresponding tissue proliferation. The tissue of LEEP group had a prolonged acute inflammatory reaction at the same time, especially the cases of secondary hemorrhage. More than 8 weeks after the surgery, both groups showed chronic inflammatory manifestations, with lymphocyte growth and corresponding tissue proliferation. CKC, cold-knife conization; LEEP, loop electrosurgical excision procedure.

bleeding. Due to the lack of the protection of the squamous epithelium, bleeding is easily caused in the process of sexual contact which also increased the frequency of genital tract inflammation. In addition, it also increases the fear of coitus and decreases the quality of sexual life. However, after the CKC operation and Sturmdorf suturing, few patients complain about the protection of the squamous epithelium.

Common cervicitis refers to mucous membrane inflammation. The cervical canal is lined by susceptible simple columnar epithelium. It is often difficult to remove pathogens due to the numerous plicas of the mucous membrane. HSIL usually invades the squamous epithelium which protects the cervix from infection. Conization of the cervix removes large areas of squamous epithelium including the transition zone and part of the columnar epithelium which inevitably leads to the shortening of the effective cervical length and absence of squamous epithelium. Thus, the postoperative residual cervical wound is exposed in the vagina with a lot of vaginal discharge after decrustation. The procedures are often associated with prolonged vaginal discharge, which is not necessarily infectious, but may be due to the healing processes and edema secondary to the procedure (19). These change the vaginal microenvironment and allow easy ascending infection. The pathogens living in the residual cervical canal often lead to chronic cervicitis. This can be seen in the results of this study. After a period of repair, there was no significant difference of the occurrence rate of abnormal leucorrhea 
between the two groups. However, the positive isolation of the pathogen from cervical secretions was different between the two groups. It was easier for pathogens to live in the cervical canal after the LEEP operation. Some studies refer to the difference of the extent of postoperative cervical hardening between the two operation methods. This might be associated with the cure of the inflammation, but further studies are needed to confirm this.

This also suggests that normal leucorrhea might not definitely mean that the pathogens had been eliminated. This study showed no correlation between the results of the leucorrhea examination and that of cervical secretion cultivation. This might be related to sampling factors. However in clinical works, it was found that some of the patients with normal leucorrhea also had pathogens or conditioned pathogens in cervical secretions after cultivation. This also explains the fact that some patients with normal leucorrhea preoperatively, had abundant capillaries and fragile tissue of the cervix which were revealed during a hysterectomy and a higher cervical infection rate after the operation. This needs to be confirmed by a multi-center study.

Judging from the results of this study, due to the thermal damage, the LEEP operation may be more likely to cause inflammatory exudation. However, in others studies, the infection condition reported that the LEEP group is superior to the CKC group. What cause such different results? Firstly, the infection rate reported did not mention what criteria were used to define the infection of abnormal secretion. Some of the studies mentioned the incidence of fever and abdominal pain. But others did not. Secondly, some reports mention the discharge change, but they did not analysis the pathogen what may cause the reproductive tract infection. From this study, there was no significant correlation between leucorrhea abnormality and cervical secretion abnormality. Thirdly, the follow-up periods of the infection are not long enough. Most of them are 2-3 weeks. Therefore, from these perspectives, this study adds some supplement for the research. The interval time of the study is more than 2 weeks. The first 2 weeks of the post-operative time, the discharge of the CKC group is more than the LEEP group, because the cervical tissue is damage into eschar by thermal. Glands secret significantly less than the CKC group. If define the abnormality of discharge as the diagnosis of infection at this time, it will conclude that the CKC procedure is more likely to cause the infection. But during the 2 to 4 weeks post-operation, eschar fall off from the wound. Due to the lack of protection of the squamous epithelium, the columnar epithelium is easier to be lived in and infected by pathogens leading to chronic cervicitis. In this study, the rate of leucorrhea abnormalities and pathogen isolated from secretions grew up, while the CKC group did not show the same trend. In the interval time between 4 and 8 weeks, the infection of LEEP group was also higher than the CKC group, it also can be due to the eschar falling off and the secondary hemorrhage beginning. We found that pathogen isolated from those cases occurred secondary hemorrhage are more than others. It may suggest that the treatment of secondary hemorrhage will prolong the duration of tissue inflammation and repair. Another reason maybe it is related to the sexual action. Some of the patients began having sex action for one and a half months after surgeries. The CKC group has the protection of the squamous epithelium because of Sturmdorf suture, while the lack of the protection, there is still a small concave in the cervical wound, and the columnar epithelium is easier to be infected.

Kietpeerakool et al. (19) searched the literature to May 2016 and found three published randomized trials involved 708 participants who had undergone excisional treatment to the cervix. As only limited data are available from three trials with overall moderate to high risk of bias, there is insufficient evidence to support use of antibiotics to reduce infectious complications following excision of the cervical transformation zone. Thus he concluded that antibiotics given for infection prevention after excision of the cervical transformation zone should only be used in the context of clinical research, to avoid unnecessary prescription of antibiotics and to prevent further increases in antibiotic resistance. But in those studies, nobody isolated the pathogen from the cervical secretion, potential and precise infections have not been effectively detected and treated. In this study, the pathogen in the CKC group was not specific, while in the LEEP group, it was mainly Escherichia coli infection. Klebsiella pneumoniae, Ureaplasma urealyticum, and Enterococcus faecalis are also found. So choosing the right antibiotics was helpful to control cervical inflammation after the operation. It also helps to control the occurrence of infection by subsequent section of the uterus.

It was found on pathological analysis that both the CKC and LEEP groups showed the different healing process. In the first two weeks after the operation, the CKC group showed an acute inflammation with increased neutrophils, tissue edema, and capillary congestion. The LEEP group marginal tissue had severe carbonation with protein degeneration. The cervix also had acute 
inflammatory reactions. Two and four weeks after the operation, tissue inflammation in the CKC group decreased to chronic condition, but in the LEEP group, the cervical eschar began to fall off, especially in the cases of secondary hemorrhage. The pathology manifestation of tissue showed edema, bleeding and inflammatory cells infiltration. Four weeks post-operation, the CKC showed chronic inflammatory manifestations with lymphocyte growth and corresponding tissue proliferation. Because of the eschar falling off and the treatment of secondary hemorrhage, the tissue of LEEP group had acute inflammatory reaction prolonged at the same time. More than 8 weeks after the surgery, both groups showed chronic inflammatory manifestations, with lymphocyte growth and corresponding tissue proliferation. The results of pathological analysis showed a consistent process of postoperative repair in the CKC and LEEP groups.

Some patients needed further treatment of a total hysterectomy based on the pathological results of cervical conization and/or their own request. However, many gynecologists feel it difficult to decide the appropriate time for the operation when the test results of vaginal secretions are normal. LRH is feasible for the treatment of cervical cancer patients with previous conization and the appropriate time interval time is after six weeks (21). Sullivan et al. (18) suggested that 6 weeks after cervical excision is associated with an increased risk for 30-day complications. Providers should consider delaying definitive surgical procedures for at least 6 weeks following excision to reduce surgical complications. This is because the cervical inflammatory factor declines to a normal level more than 6 weeks after cervical conization.

\section{Study limitations}

One limitation of this study is that the number of cases is not enough, especially the lack of information on whether the changes of vaginal microecology affect the infection of patients pre- and pro-operation. It will have more clinical significance. The second limitation is not to consider whether the patients' habits, such as sport, diet, etc. Those factors may be related to the postoperative secondary bleeding, because the patients may have ethnic and regional differences. This study is also limited by its single-centre design. These data do reflect practice in one center, but other centers or clinicians may differ in practice or use different criteria to judge. Further research to deepen understanding of these factors can provide opportunities to improve clinical practice.

\section{Conclusions}

In conclusion, this study indicates that the chance of persistent cervical infection after the CKC operation with Sturmdorf suturing is lower than that of after LEEP surgery, with the former being superior to the latter. Although the CKC operation is an old technology, it is still worth extending. Both methods have the time for recovery and inflammation susceptibility. Clinicians can pay attention to the corresponding examination and treatment according to their characteristics. Postoperative cervical secretions culture and drug susceptibility tests are helpful for finding hidden pathogens, shortening the time of inflammation reaction for the following treatment. In addition, especially for patients with a subsequent hysterectomy, removal of the cervical pathogens and the elimination of inflammation will be conducive to control subsequent surgical infections.

\section{Acknowledgments}

Funding: This work was supported by Science Foundation of Fujian Province (2015J01419).

\section{Footnote}

Conflicts of Interest: All authors have completed the ICMJE uniform disclosure form (available at http://dx.doi. org/10.21037/tcr.2019.12.34). The authors have no conflicts of interest to declare.

Ethical Statement: The authors are accountable for all aspects of the work in ensuring that questions related to the accuracy or integrity of any part of the work are appropriately investigated and resolved. The study was conducted in accordance with the Declaration of Helsinki (as revised in 2013). All protocols and informed consent procedures were approved by the ethics committee of the hospital (No. 2017034), and informed written consent was obtained from each woman.

Open Access Statement: This is an Open Access article distributed in accordance with the Creative Commons Attribution-NonCommercial-NoDerivs 4.0 International License (CC BY-NC-ND 4.0), which permits the noncommercial replication and distribution of the article with the strict proviso that no changes or edits are made and the original work is properly cited (including links to both the formal publication through the relevant DOI and the license). 
See: https://creativecommons.org/licenses/by-nc-nd/4.0/.

\section{References}

1. Santesso N, Mustafa RA, Schünemann HJ, et al. World Health Organization Guidelines for treatment of cervical intraepithelialneoplasia 2-3 and screen-and-treat strategies to prevent cervical cancer. Int J Gynaecol Obstet 2016;132:252-8.

2. Prendiville W, Cullimore J, Norman S. Large loop excision of the transformation zone (LLETZ). A new method of management for women with Squamous Intraepithelial Lesion. Br J Obstet Gynaecol 1989;96:1054-60.

3. Wright TC Jr, Gagnon S, Richart RM, et al. Treatment of Squamous Intraepithelial Lesion using the loop electrosurgical excision procedure. Obstet Gynecol 1992;79:173-8.

4. Lindeque BG. Management of cervical premalignant lesions. Best Pract Res Clin Obstet Gynaecol 2005;19:545-61.

5. Basu P, Taghavi K, Hu SY, et al. Management of cervical premalignant lesions. Curr Probl Cancer 2018;42:129-36.

6. Mathevet P, Dargent D, Roy M, et al. A randomized prospective study comparing three techniques of conization: cold knife, laser, and LEEP. Gynecol Oncol 1994;54:175-9.

7. Duggan BD, Felix JC, Muderspach L, et al. Cold-knife conization versus conization by the loop electrosurgical excision procedure: a randomized, prospective study. Am J Obstet Gynecol 1999;180:276-82.

8. Takac I, Gorisek B. Cold knife conization and loop excision for Squamous Intraepithelial Lesion. Tumori 1999;85:243-6.

9. Bozanović T, Ljubic A, Momcilov P, et al. Cold-knife conization versus the loop electrosurgical excision procedure for treatment of cervical dysplasia. Eur J Gynaecol Oncol 2008;29:83-5.

10. El-Nashar SA, Shazly SA, Hopkins MR, et al. Loop electrosurgical excision procedure instead of coldknife conization for Squamous Intraepithelial Lesion in women with unsatisfactory colposcopic examinations: a systematic review and meta-analysis. J Low Genit Tract Dis 2017;21:129-36.

11. Jiang YM, Chen CX, Li L. Meta-analysis of cold-knife conization versus loop electrosurgical excision procedure for cervical intraepithelial neoplasia. Onco Targets Ther 2016;9:3907-15.

12. Janthanaphan $M$, Wootipoom $V$, Tangsinmunkong $K$, et al. Comparison of success rate and complications of contourloop excision of the transformation zone (C-LETZ) with cold knife conization (CKC) in high grade lesion (HGL) from colposcopic impression. J Med Assoc Thai 2009;92:1573-9.

13. Kietpeerakool C, Srisomboon J, Suprasert P, et al. Routine prophylactic application of Monsel's solution after loop electrosurgical excision procedure of the cervix: is it necessary? J Obstet Gynaecol Res 2007;33:299-304.

14. Borbolla Foster A, Symonds I. A comparative study of efficacy and outcomes of large loop excision of the transformation zone procedure performed under general anaesthesia versus local anaesthesia. Aust N Z J Obstet Gynaecol 2012;52:128-32.

15. Mints $M$, Gaberi V, Andersson S. Miniconization procedure with C-LETZ conization electrode for treatment of cervical intraepithelial neoplasia: a Swedish study. Acta Obstet Gynecol Scand 2006;85:218-23.

16. Mitchell MF, Tortolero-Luna G, Cook E, et al. A randomized clinical trial of cryotherapy, laser vaporization, and loop electrosurgical excision for treatment of squamous intraepithelial lesions of the cervix. Obstet Gynecol 1998;92:737-44.

17. Perlman SE, Lubianca JN, Kahn JA. Characteristics of a group of adolescents undergoing loop electrical excision procedure (LEEP). J Pediatr Adolesc Gynecol 2003;16:15-20.

18. Sullivan SA, Clark LH, Staley AS, et al. Association between timing of cervical excision procedure to minimally invasive hysterectomy and surgical complications. Gynecol Oncol 2017;144:294-8.

19. Kietpeerakool C, Chumworathayi B, Thinkhamrop J, et al. Antibiotics for infection prevention after excision of the cervical transformation zone. Cochrane Database Syst Rev 2017;1:CD009957.

20. Song T, Seong SJ, Kim BG. Regeneration Process After Cervical Conization for Cervical Intraepithelial Neoplasia. Obstet Gynecol 2016;128:1258-64.

21. Li H, Jang JY, Li H, et al. The influence of interval between conization and laparoscopic radical hysterectomy on the morbidity of patients with cervical cancer. Eur J Gynaecol Oncol 2012;33:601-4.

Cite this article as: Cai L, Huang Y, Lin C, Liu G, Mao X, Dong B, Lu T, Sun P. A comparison study of postoperative infection analysis of cold-knife conization and loop electrosurgical excision procedure for cervical highgrade squamous intraepithelial lesion. Transl Cancer Res 2020;9(2):949-957. doi: 10.21037/tcr.2019.12.34 